\title{
Impact of Microscopic NAPL-Water Interface Configurations on Subsequent Gas Injection into Water-Wet Permeable Rocks
}

\author{
Ahmed Al-Futaisi ${ }^{\text {a* }}$ and Tad W. Patzek ${ }^{\mathrm{b}} \dagger$ \\ ${ }^{a}$ Civil and Architectural Engineering, \\ Sultan Qaboos University, Alkhoudh 123, Oman \\ ${ }^{\mathrm{b}}$ Department of Civil and Environmental Engineering, \\ UC Berkeley, CA 94720, USA
}

Predictive field-scale models of the concurrent flow of three fluids require accurate predictions of five macroscopic flow descriptors: three relative permeabilities and two capillarypressures as functions of the fluid saturations and saturation history. Since direct measurement of these descriptors is very difficult, and empirical correlations are often unreliable, the use of physically-based pore-scale models has become an appealing alternative. In this paper, we describe the features of our quasi-static pore network model for three immiscible fluids. The model integrates a realistic representation of pore connectivity and morphology reconstructed from 3D micro-focused X-ray CT images, a realistic description of fluid displacement mechanisms, and a sound representation of the wetting properties of the rock. All pore-level displacement mechanisms: piston-type, snap-off, cooperative pore-body filling, and double-displacements are considered with arbitrary contact angles and spreading coefficients. The proposed model is used to simulate gas injection into water-wet Bentheimer sandstone that initially contains water and NAPL after two-phase drainage followed by twophase imbibition. The gas injection is performed using a cluster-based invasion percolation algorithm with trapping.

\section{Introduction}

Three-phase flow in porous media is important in many cases: gas injection and thermal flooding in oil reservoirs, flow of non-aqueous phase liquids (NAPL) in the vadose zone, vapor extraction in soil, radio-nuclide migration from hot repositories of nuclear waste, etc.. Predictive field-scale models of the concurrent flow of three fluids require accurate predictions of the macroscopic relative permeabilities, capillary pressures, and spacial distributions of trapped fluids. Experiments with three-phase flow are difficult, expensive, and time consuming. Furthermore, it is impractical to measure relative permeabilities for all possible saturation paths that may occur in a three-fluid flow system. Instead, empirical correlations of three-phase relative permeabilities and capillary pressures have been obtained from the interpolations of two-phase, oil-water and oil-gas, flow experiments [1-3]. The empirical

\footnotetext{
${ }^{*}$ Corresponding author: futaisi@squ.edu.om , Address: P.O. Box 33, Alkhoudh 123, Oman

†patzek@patzek.berkeley.edu.
} 
nature and incomplete physics of these three-phase models [4] render them insufficient to predict the general three-phase flow behavior.

A physically-based three-phase pore network model is an appealing alternative to the empirical correlations of measured relative permeabilities and capillary pressures [5-7]. Common to all these three-phase pore network models is that they have been performed on regular 2D or 3D lattices. Only Lerdahl et al. [8] used a reconstruction algorithm based on thin-section analysis to generate a topologically disordered network. Their pore network model illustrated a very promising comparison between the model and experimental three-phase relative permeabilities on Berea sandstone during gas injection after two-phase primary drainage $[9,10]$. However, their model has not included gas injection after twophase secondary imbibition in which oil exists as many trapped clusters. Recently, Piri and Blunt [11] have also developed a three-phase flow model on the same network constructed by Lerdahl et al. [8]. Piri \& Blunt's pore network model has ignored double and multiple displacements; they only considered gas injection for relatively high oil saturations, and with little or no oil initially trapped. Here, we attempt to reproduce the Lerdahl et al. [8] model and extend it to simulate gas injection after secondary imbibition.

\section{The Network and Two-Phase Flow Model}

The pore network we use $[12-15]$ is a realistic representation of a sample of Bentheimer sandstone reconstructed from the $3 \mathrm{D}$ micro-focused X-ray CT image. The network is a courtesy of Statoil Company. It consists of cylindrical ducts with angular cross-sections. The complex topology and geometry of the network is detailed in $[12,16]$. The two-phase flow model is quasi-static, i.e., capillary forces dominate, gravity is accounted for, and the effects of viscous forces are ignored. The flow simulations proceed in the following sequence: (1) fully saturate the network with water, (2) perform primary drainage (i.e., oil displaces water) up to a predefined maximum capillary pressure, (3) alter wettability, and (4) perform secondary imbibition (i.e., water displaces oil). Capillary pressure curves are calculated using percolation algorithms [14] with piston-type, snap-off, and cooperative pore-body filling mechanisms $[12,16]$. Relative permeabilities are calculated using Darcy's law and accurate expressions of the hydraulic conductance of phases bulk flow [13], corner flow [17], and intermediate layers flow [18]. Spatial distribution of oil clusters are identified using the Hoshen-Kopelman algorithm [19] extended by us to disordered networks [15].

\section{Assumptions for the Three-Phase Flow Model}

More detailed description of the physics of three-phase flow is yet to be uncovered. However, great deal of it have been recently recognized from micromodels and other experiments. Here, we present and discuss the physics and main assumptions implemented in our threephase flow model. The discussion includes the spreading coefficient, three-phase contact angles, displacement mechanisms, capillary entry pressures, and saturations paths.

\subsection{Spreading Coefficient and Admissible Three-Phase Contact Angles}

An important characteristic of three-phase flow is the existence of layers of the intermediate fluid sandwiched between the two other fluids. For example, in Figure 2G, the intermediate layers of oil are sandwiched between the water that resides in the corners of the pore and 
gas which occupies the center of the pore. It is believed that drainage of these layers is responsible for the high oil recoveries seen experimentally [20,21]. The existence or absence of such oil layers is related to the equilibrium spreading coefficient, contact angles, capillary pressures, and pore geometry $[18,22-24]$. The question that immediately arises is what possible values of contact angles one should assign? There is yet no direct answer to this question. For specific systems, there have been attempts to relate contact angles to the interfacial tensions and spreading coefficient $[8,25,26]$. In our simulator, similar to Lerdahl et al. [8], $\theta_{o w}, \theta_{g o}$, and $\theta_{g w}$ are all defined randomly from a uniform probability distribution function, scaled to different ranges. We believe that this is the best we can do at this stage until the quantitatively new theories of three fluid phase-solid wetting behavior appear.

\subsection{Initial Conditions}

We restrict this study to the initial condition that corresponds to a network of water and oil with a specific initial water saturation, $S_{w_{i}}$, and specific initial o/w configuration. The network initial o/w configurations are set by the oil-water drainage or imbibition processes and the last $\mathrm{o} / \mathrm{w}$ capillary pressure attained during each of these two processes. After primary drainage of an initially water-wet rock, oil exists as one cluster that may or may not span the network depending on the maximum capillary pressure attained during the oil invasion. In contrast, after secondary imbibition, oil exists as many disconnected clusters. Our results indicate that the number and spacial distribution of the trapped oil clusters has a considerable impact on the three-phase flow characteristics.

\subsection{Fluid Configurations}

At the end of three-phase primary drainage or secondary imbibition, and depending on $\mathrm{o} / \mathrm{w}$ contact angle, the water and oil configuration in a duct is one of the four possible configurations depicted in Figure 1. Configuration A is a duct completely filled with water, B is a duct with water in the corners and oil in the center after primary drainage or spontaneous imbibition, $\mathrm{C}$ is the same as $\mathrm{B}$ but after forced imbibition, and $\mathrm{D}$ is a duct with water in the corners and the center and oil in the intermediate layers after forced imbibition with extreme $\mathrm{o} / \mathrm{w}$ contact angles. When gas is introduced into the system, the displacement is always piston-type advance because gas is not initially present in the system and it is non-wetting. Therefore, gas will only occupy the center of the pore. Hui and Blunt $[26,27]$ showed that gas invasion into the o/w configurations shown in Figure 1 results into five additional g/o/w configurations, Figure 2. Details of these fluid configurations and their threshold capillary entry pressures are described and defined in $[8,27]$. In this analysis, we assume that a fluid-water interface is pinned along the boundary of the region of altered wettability. This interface adjusts its curvature until it reaches the new prescribed contact angle. We now assume that the new receding contact angle is larger and, therefore, water can be displaced completely from the corner if $\theta+\beta_{i} \geq \pi / 2$. The phase saturations and conductances are calculated using the procedures described in [14] and [18].

\subsection{Fluid Clusters and Their Displacement Mechanisms}

At the end of the two-phase flow (primary drainage or secondary imbibition), oil exists in four types of clusters: (I) clusters spanning the network, (II) clusters connected to the inlet but disconnected from the outlet, (III) clusters connected to the outlet but disconnected from the inlet, and (IV) clusters disconnected from the inlet and outlet. The oil clusters 


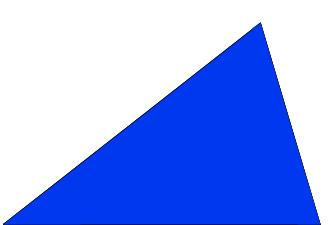

A

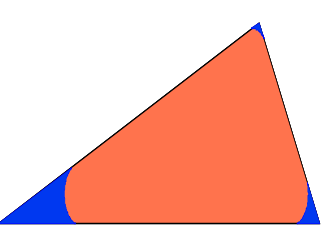

B

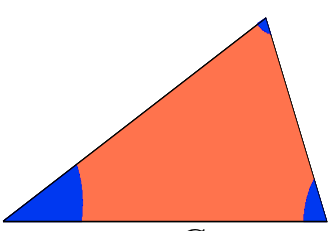

C

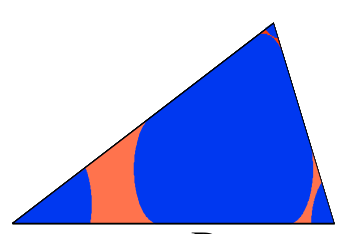

D

Figure 1. Possible $o / w$ configurations in a duct after the secondary imbibition ends (water is dark and oil is gray)

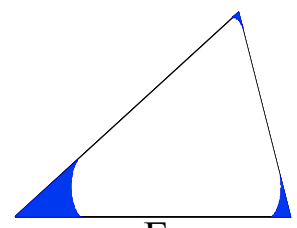

E

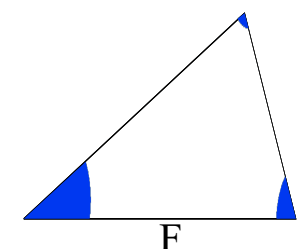

F

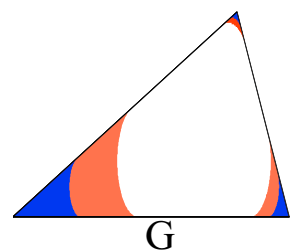

G

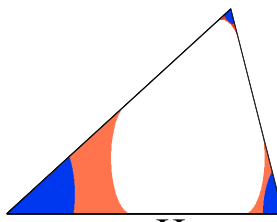

$\mathrm{H}$

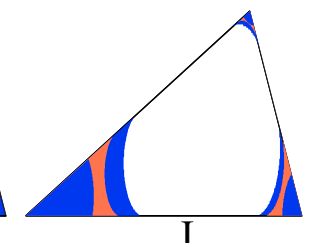

Figure 2. Possible $g / o / w$ configurations in a duct during gas invasion (water is dark, oil is gray, and gas is white)

that are connected to the outlet face (I and III) are directly displaced by gas and collected at the outlet. This process is called direct oil displacement. In contrast, the oil clusters that are not connected to the outlet (I and IV) can only be displaced by multiple displacement mechanisms. In case of the first gas injection into the system, micromodel experiments [2830] have shown that the necessary multiple displacement is double drainage in which gas displaces oil and oil displaces water. van Dijke et al. [30] have observed higher-order multiple displacements in the case of alternate cycles of gas and water injection. In general, one must consider all possible multiple displacements using a minimum path algorithm, and find the displacement sequence that results in the minimum capillary pressure. However, such a procedure is very demanding computationally when considering large, disordered networks. Our study is restricted to the first gas injection into the network, therefore it is sufficient to consider only the double drainage displacement mechanism. Incorporation of the double drainage displacement is quite difficult for two reasons: (1) tracking the boundaries of the moving trapped clusters, and (2) conserving the oil volume. These two problems are especially challenging in the network considered in this analysis because of its complex geometry and connectivity.

\section{Case Study}

Here we present the results of different gas injection simulations after o/w primary drainage and secondary imbibition. All simulations have been performed with equilibrium interfacial tensions equal to $35 \times 10^{-3} \mathrm{~N} / \mathrm{m}$ for water, $25 \times 10^{-3} \mathrm{~N} / \mathrm{m}$ for oil, and $50 \times 10^{-3} \mathrm{~N} / \mathrm{m}$ for gas. These values indicate that the rock spreading coefficient is $-10 \mathrm{mN} / \mathrm{m}$. The water, oil, and 


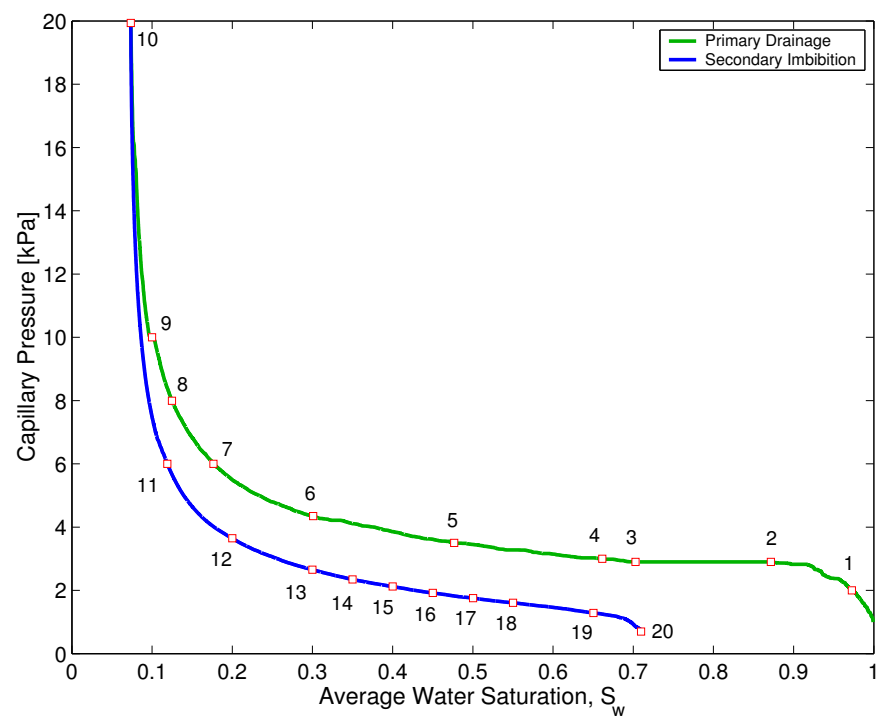

Figure 3. Gas injection after $o / w$ primary drainage or secondary imbibition with different initial saturations (denoted by the squares)

gas densities are 1000,950 , and $1.2 \mathrm{~kg} / \mathrm{m}^{3}$, respectively. The $\mathrm{o} / \mathrm{w}$ primary receding contact angles during primary drainage, $\theta_{o w}$, are $0^{\circ}$ to $10^{\circ}$. The $\mathrm{o} / \mathrm{w}$ advancing contact angles during secondary imbibition, $\theta_{\text {ow }}$, are $10^{\circ}$ to $30^{\circ}$. The o/w secondary receding contact angles during gas injection, $\theta_{\text {ow }}$ are $10^{\circ}$ to $30^{\circ}$. The g/o receding contact angles, $\theta_{\text {go }}$, and $\mathrm{g} / \mathrm{w}$ receding contact angles, $\theta_{g w}$, during gas injection are $0^{\circ}$ to $10^{\circ}$. Notice that the values of these contact angles determine that the rock is water-wet. The maximum capillary pressure in primary drainage, $P_{c, o w}^{\max }$, and gas injection, $P_{c, g w}^{\max }$, is $188000 \mathrm{~Pa}$. For the relative permeability calculations, we assume $1.0 \times 10^{-3} \mathrm{~Pa}$-s for the water and oil viscosities, and $1.0 \times 10^{-4} \mathrm{~Pa}$-s for the gas viscosity. The $\mathrm{o} / \mathrm{w}$ interface boundary condition is assumed no-slip, whereas the $\mathrm{g} / \mathrm{o}$ and $\mathrm{g} / \mathrm{w}$ interface boundary condition is perfect-slip. The two-phase (water and oil) primary drainage and secondary imbibition capillary pressure curves are plotted in Figure 3. Gas injection simulations are performed with 20 different initial o/w saturations and interface configurations along these two curves. There are 10 simulations along the primary drainage curve, and 10 along the secondary imbibition curve. As mentioned earlier, after primary drainage, oil exists as a single cluster. After secondary imbibition, oil exists as many clusters.

\section{Results of Gas Injection Simulations}

Figure 4 compares the saturation paths obtained from both saturation histories. It is observed that the saturation paths obtained after primary drainage (Figure 4a) are more scattered, and those after secondary imbibition (Figure 4a) are less. Moreover, for gas injection after secondary imbibition the different saturation paths converge. In both cases, the trapped oil saturation reaches very small values, close to $1 \%$, due to our assumption that one intermediate oil layer per duct is enough to maintain the local oil connectivity. In 


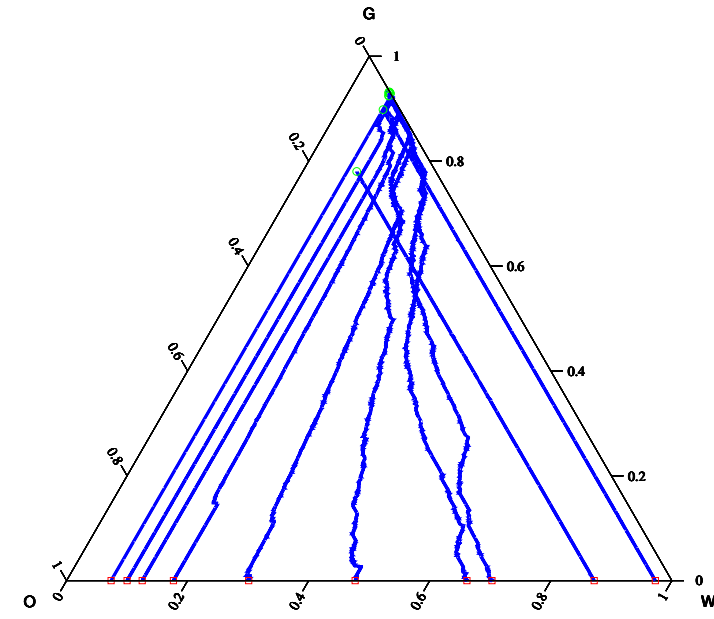

(a) gas injection after primary drainage

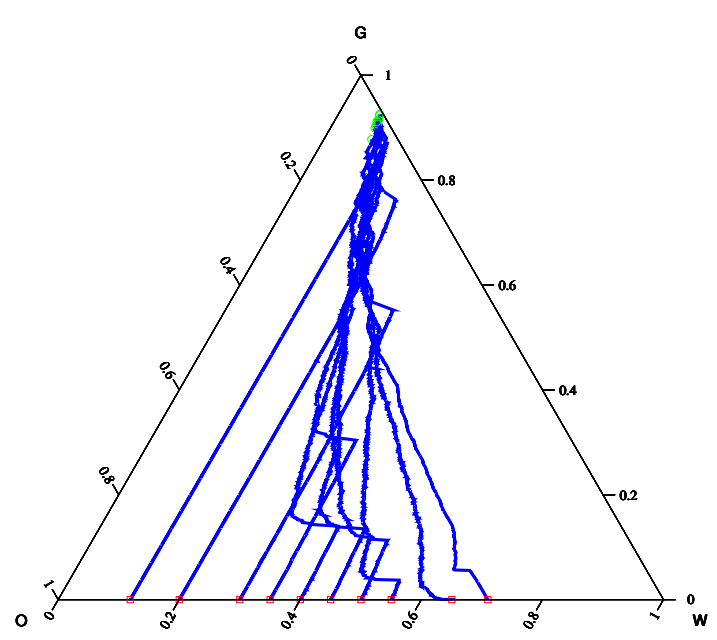

(b) gas injection after secondary imbibition

Figure 4. All saturation paths during gas injection with different initial water saturations and different two-phase displacement processes

reality, connectivity of the intermediate oil layers is more complicated and tenuous. Figure 5 summarizes the invasion events in both sets of simulations (Figure 5a for primary drainage and Figure 5b for secondary imbibition) as functions of the initial water saturation, $S_{w_{i}}$, at the onset of gas injection. As $S_{w_{i}}$ increases, direct oil decreases, direct water increases, and double drainage increases. As discussed before, the double drainage mechanism affects more gas injection after secondary imbibition because of the large number of trapped oil clusters.

Figure 6 compares the water, oil, and gas relative permeabilities plotted against their own saturations after the primary drainage (Figure 6a) and secondary imbibition (Figure 6b) simulations. Although our calculations are for a different sandstone, the relative permeability plots after primary drainage are in good qualitative agreement with the Statoil simulations of gas injection into Berea sandstone [8]. In the case of gas injection after secondary imbibition, one can immediately observe that the scatter of the relative permeabilities of oil is significantly larger than those of gas and water. This result demonstrates that the oil permeability in three-phase flow strongly depends on the saturation history. The water relative permeability is the least scattered indicating that it does not depend on the saturation history, i.e., it is only a function of its own saturation. The gas relative permeability has noticeable scatter especially at low gas saturations. Furthermore, it is observed that the oil permeability scatter after secondary imbibition is more severe than after primary drainage. We attribute this increased scatter to the internal movements and joining of the trapped oil clusters.

\subsection{Discussion of Results}

We begin with gas injection after primary drainage of water by oil. With high water saturations at the onset of gas injection $\left(S_{w_{i}}=0.97\right.$ and 0.87$)$, the invading oil cluster does not reach the outlet, and the major displacement process is direct water. There are no direct 


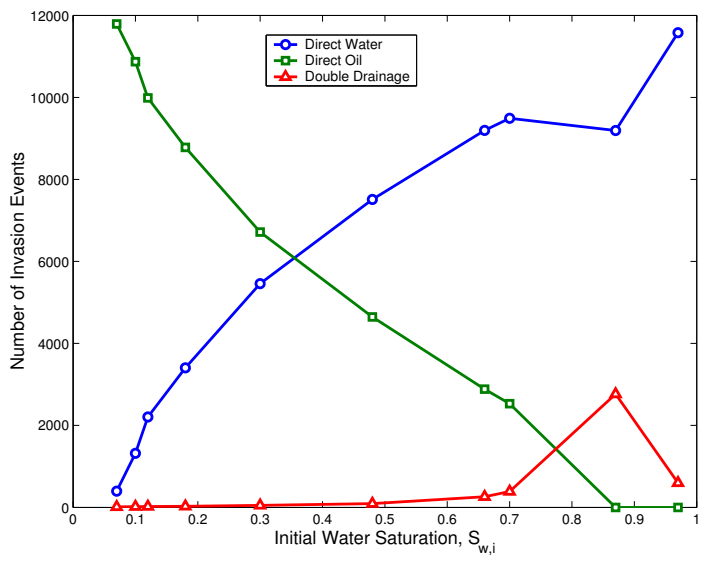

(c) gas injection after primary drainage

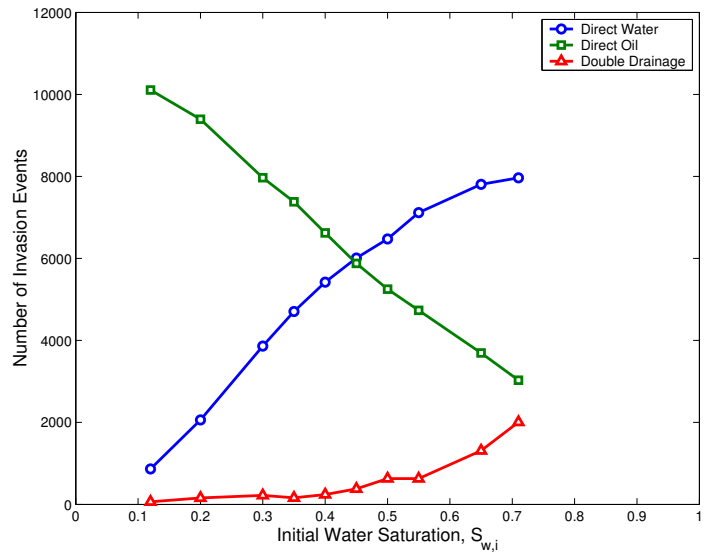

(d) gas injection after secondary imbibition

Figure 5. Direct water, direct oil, and double drainage events as function of initial water saturation from all gas injection simulations

oil displacements. Some double drainage displacements are observed, but they do not succeed in moving oil all the way to the outlet face. Thus, oil saturation remains unchanged and its relative permeability is zero since the oil has not formed a spanning cluster. Assigning zero to the relative permeability of oil is somewhat misleading, since the oil is actually moving inside the network. The incorporation of the transient oil clusters into the oil relative permeability is impossible within the quasi-static framework of this analysis.

As the maximum capillary pressure in primary drainage increases, the relative permeability to gas also increases, and the water relative permeability decreases. Water relative permeability becomes very small as the water begins to flow predominantly in the continuous filaments along the duct corners. At lower water saturations, $S_{w_{i}}=0.70$ to 0.48 , oil breakthrough occurs in two-phase primary drainage. Thus a network-spanning oil cluster is formed. Smaller oil clusters connected to the inlet face, but not to the outlet, may also be formed. In such cases, direct oil displacement is more favored than double drainage displacements. Therefore, it is observed that the number of direct oil displacements gradually increases and the number of double drainage displacements decreases. In general, the importance of direct water displacement decreases; however, direct water remains the major displacement mechanism because of the relatively high water saturation at the onset of gas injection.

Oil relative permeability decreases in a manner similar to that of water because the oil is allowed to flow in the intermediate layers, or oil filaments, in contact with the corner water filaments. However, it is observed that the oil relative permeability curves are not as smooth as those of water, because of the possible reconnection of oil clusters. For water saturations less than 0.30 , oil exists in a large cluster connecting the inlet and outlet faces of the network. Therefore, double drainage displacements almost cease and the number of direct oil displacements exceeds that of direct water displacements. The gas and oil relative permeabilities are now similar to those in two-phase flow. The water relative permeabilities 

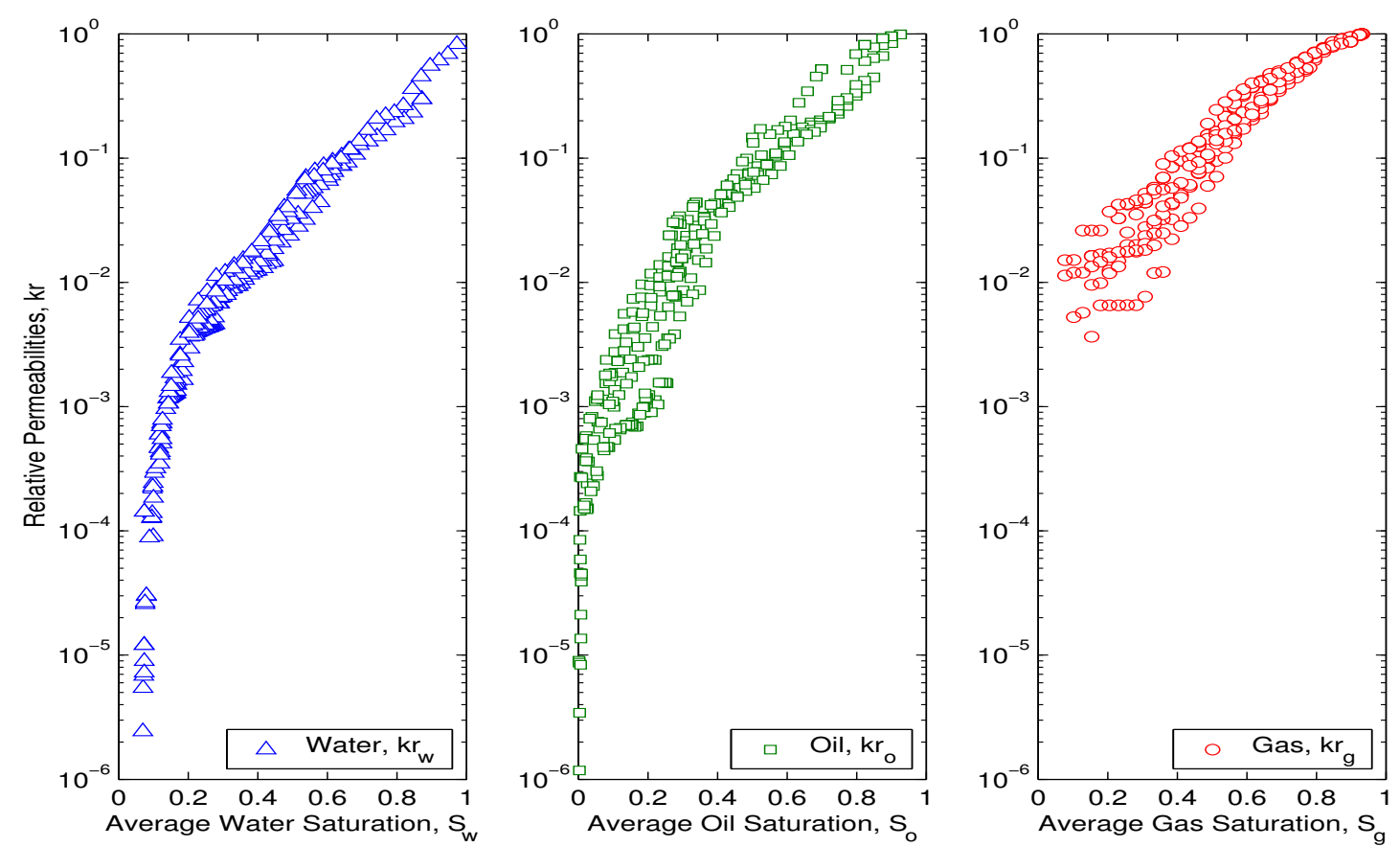

(a) gas injection after primary drainage
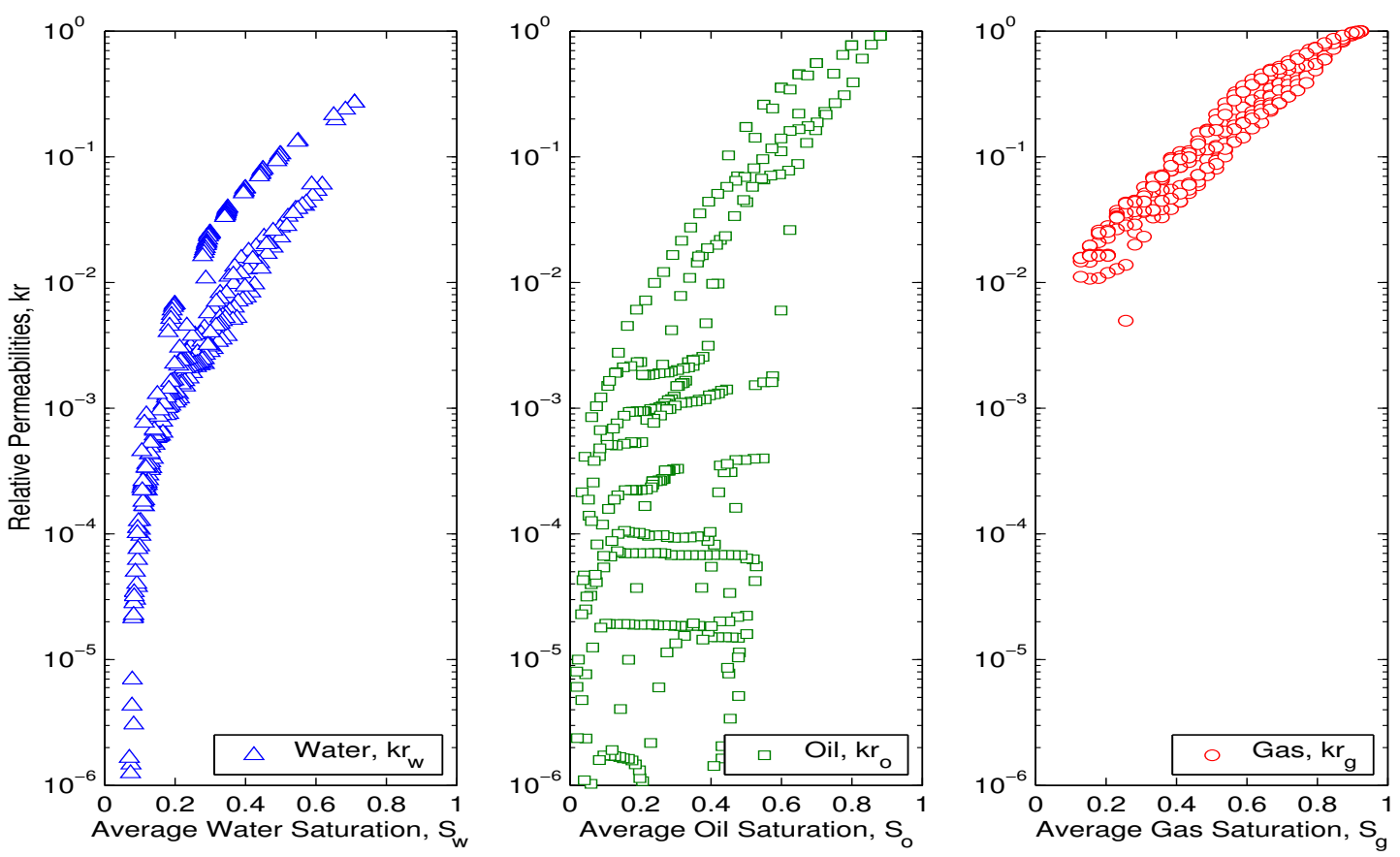

(b) gas injection after secondary imbibition

Figure 6. Water, oil, and gas relative permeabilities as functions of their saturations for all gas injection simulations 
are very small because water flows entirely in the corner filaments.

During secondary imbibition displacement, the spanning oil cluster created during primary drainage is broken-up into smaller clusters by the bond-breaking mechanisms, such as snapoff and cooperative pore-body filling, e.g., [12]. It is expected that as secondary imbibition proceeds, more and more disconnected oil clusters are created. As a result, double drainage displacements enter the picture again and define the flow behavior. However, it is observed from our simulations that direct oil displacements are more favored than double drainage. Thus, initially the flow follows a saturation path with constant water saturation. Once further direct oil displacements become difficult, the frequency of double drainage increases, and water saturation goes down. One can also observe, as the initial water saturation increases, the number of direct water displacements increases due to the large amount of water in the network. For example, when $S_{w_{i}}=0.65$ to 0.71 at the onset of gas injection, the water saturation decreases and the oil saturation remains almost constant. Oil is produced when the moving trapped clusters reach the outlet face. We note that the o/w capillary pressure increases in steps whenever a trapped oil cluster is displaced. We also note that the water and oil relative permeabilities have wider variability than the cases of gas injection after primary drainage. Moreover, the oil relative permeabilities increase in large steps whenever a trapped oil cluster connects with a spanning cluster. We call this effect the "weeping" oil relative permeability. A similar weeping effect was observed experimentally in the flow of oil emulsions in water. The relatively large magnitude of step increases of the oil permeability is attributed to the small size of the network.

These observations on the phase relative permeabilities highlight the importance of not only the values of the initial two-phase saturations, but also of the initial fluid configurations exemplified by the respective cluster distributions. This conclusion is further illustrated by the fluid iso-permeability contours, or "isoperms," in Figure 7. These isoperms are linearly interpolated to preserve their actual shapes and avoid smoothing. The water isoperms, in most cases, depend only on water saturation. However, there are some situations, especially after secondary imbibition, where this assumption fails. The gas isoperms are clearly curved and convex up. Therefore, the gas permeability cannot be described by its own saturation only. Finally, the oil isoperms definitely depend on two saturations.

\section{Conclusions}

- A complete quasi-static model of drainage of oil and water by gas in water-wet rocks has been developed. The model calculates the three fluid saturation paths, capillary pressures, and relative permeabilities. The model incorporates current state-of-the-art description of pore-scale displacement mechanisms and wettability.

- Our calculations clearly demonstrate that the saturation paths in three-phase drainage are very sensitive to the initial conditions, and depend on the prior saturation history. Therefore, pore network models should be used in the continuum three-phase flow simulators to generate the saturation-history-dependent relative permeabilities and capillary pressures.

- The trapped oil saturation after gas injection is very small, about $1 \%$, consistent with our assumption that a single intermediate oil layer in a duct is enough to maintain the 


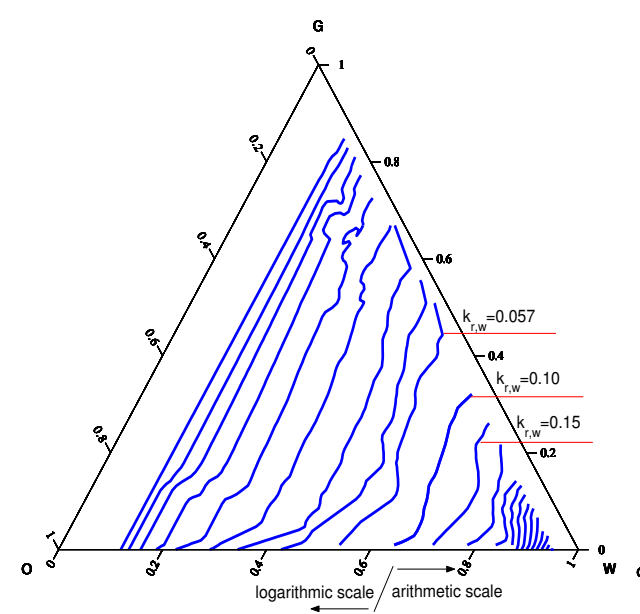

(a) water isoperms

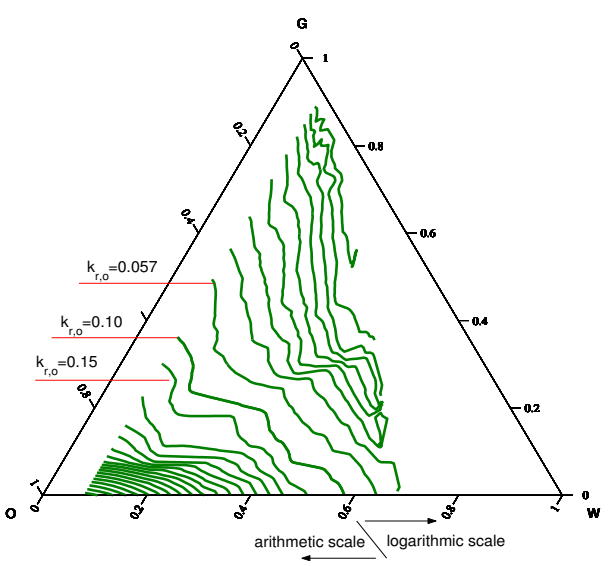

(c) oil isoperms

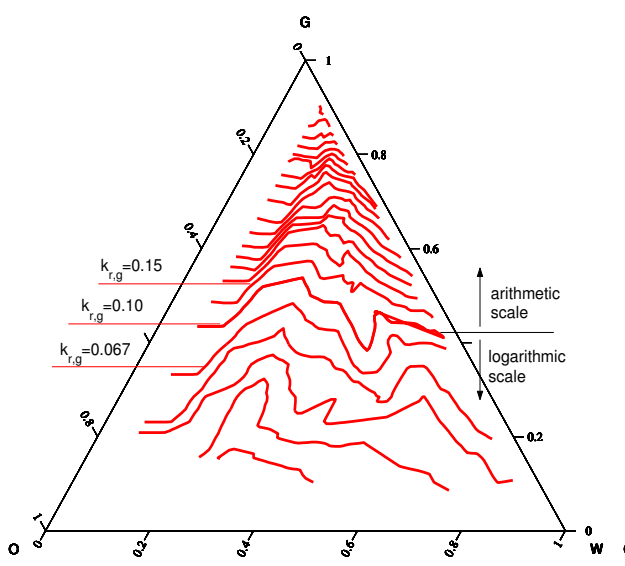

(e) gas isoperms

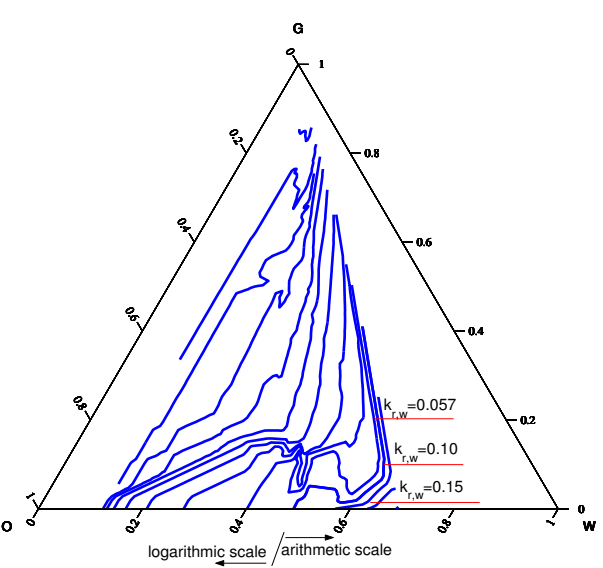

(b) water isoperms

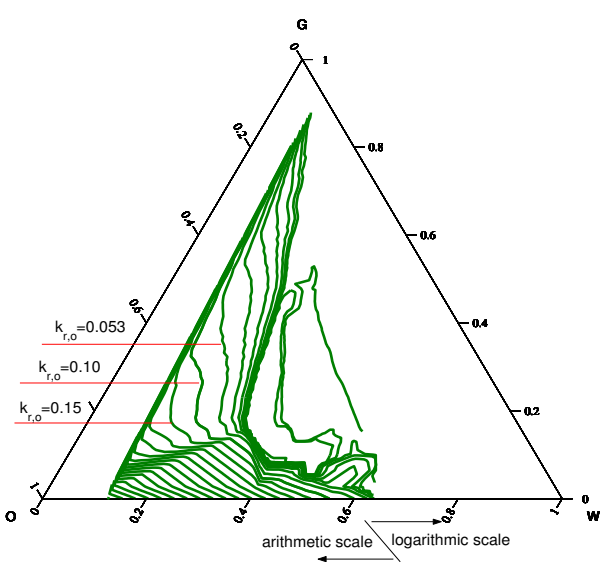

(d) oil isoperms

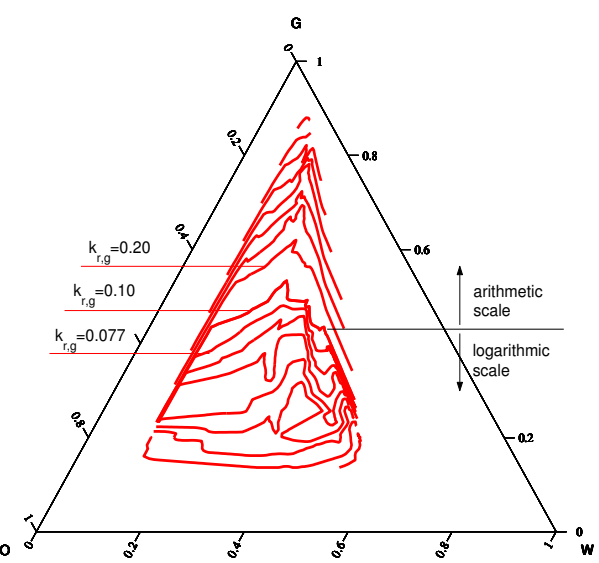

(f) gas isoperms

Figure 7. Water, oil, and gas isoperms for gas injection after primary drainage (left) and secondary imbibition (right) 
oil connectivity. This assumption is perhaps too optimistic and requires further study.

- The scatter of the three-phase saturation paths following primary drainage is wider than that after secondary imbibition. The different saturation paths converge, indicating that at high gas saturation the prior history is forgotten.

- The water isopermeability contours, or "isoperms," usually depend only on the water saturation. However, there are some instances of secondary imbibition, where these isoperms depend on two saturations. The gas isoperms are always curved. Therefore, gas permeability cannot be described by its own saturation only. Finally, the oil permeability definitely depends on two saturations, exhibiting a wide scatter when plotted against just the oil saturation.

\section{REFERENCES}

1. H. L. Stone. Estimation of three-phase relative permeability and residual oil data. J. Canad. Petrol. Tech., 12:53-61, 1973.

2. J. C. Parker and R. J. Lenhard. Determining three-phase permeability-saturationpressure relations from two-phase measurments. J. Petro. Sci. Engin., 4:57-65, 1990.

3. M. J. Blunt. An empirical model for three-phase relative permeability. SPE 56474: Proceedings of the SPE Annual Technical Conference and Exhibition, Houston, TX, pages 3-6, October, 1999.

4. R. Juanes and T. W. Patzek. Relative permeabilities for strictly hyperbolic models of three-phase flow in porous media. Trans. Porous Media, 2002. (Submitted).

5. A. A. Heiba and H. T. Davis L. E. Scriven. Statistical network theory of three-phase relative permeabilities. SPE 12690. Proceeding of the 1984 4th SPE/DOE Symposium on Enhanced Oil Recovery, Tulsa, Oklahoma, 1984.

6. W. E. Soll and M. A. Celia. A modified percolation approach to simulating three-fluid capillary pressure-saturation relationships. Adv. Water Resour., 16(2):107-126, 1993.

7. D. H. Fenwick and M. J. Blunt. Network modeling of three-phase flow in porous media. Soci. Petro. Engrg. J., 3:86-97, 1998.

8. T. R. Lerdahl, P. E. Øren, and S. Bakke. A predictive network model for three-phase flow in porous media. SPE 59311: Proceedings of the SPE/DOE Symposium on Improved Oil Recovery, Tulsa, OK, pages 2-5, April, 2000.

9. M. J. Oak, L. E. Baker, and D. C. Thomas. Three-phase relative permeability of Berea sandstone. J. Petro. Tech., 42(8):1054-1061, August 1990.

10. M. J. Oak. Three-phase relative permeability of intermediate-wet Berea sandstone. In SPE Annual Technical Conference and Exhibition, Dallas, TX, October 6-9, 1991. (SPE 22599).

11. M. Piri and M. J. Blunt. Pore-scale modeling of three-phase flow in mixed-wet systems. Proceedings of the SPE Annual Technical Conference and Exhibition, San Antonio, TX, September, 2002.

12. T. W. Patzek. Verification of a complete pore network simulator of drainage and imbibition. Soci. Petro. Engrg. J., 6:144-156, 2001.

13. T. W. Patzek and D. B. Silin. Shape factor and hydraulic conductance in noncircular capillaries: I. One-phase creeping flow. J. Colloid Interface Sci., 236(2):295-304, 2001. 
14. A. Al-Futaisi and T. W. Patzek. Impact of wettability alteration on two-phase flow characteristics of sandstone - A quasi-static model. Water Resour. Res., 39(2):314-324, 2003.

15. A. Al-Futaisi and T. W. Patzek. Extension of the Hoshen-Kopelman algorithm to a non-lattice environments. Physica A, 321:665-678, 2003.

16. P. E. Øren, S. Bakke, and O. I. Arntzen. Extending predictive capabilities to network models. Soci. Petro. Engrg. J., 3(4):324-336, 1998.

17. T. W. Patzek and J. D. Kristensen. Shape factor and hydraulic conductance in noncircular capillaries: II. Two-phase creeping flow. J. Colloid Interface Sci., 236(2):305-317, 2001.

18. A. Al-Futaisi and T. W. Patzek. Three-phase hydraulic conductances in angular capillaries. SPE 75193. Proceedings of the 13th SPE/DOE Symposium on Improved Oil Recovery, Tulsa, Oklahoma, 2002.

19. J. Hoshen and R. Kopelman. Percolation and cluster distribution I. Cluster multiple labeling technique and critical concentration algorithm. Phys. Rev. B, 14(8):3438-3445, 1976.

20. J. M. Dumoré and R. S. Schols. Drainage capillary functions and the influence of connate water. Soci. Petro. Engrg. J., 14:437-444, 1974.

21. D. Zhou and M. J. Blunt. Effect of spreading coefficient on the distribution of light non-aqueous phase liquid in the subsurface. J. Contam. Hydrol., 25:1-19, 1997.

22. M. Dong, F. A. Dullien, and I. Chatzis. Imbibition of oil in film form over water present in edges of capillaries with an angular cross-section. J. Colloid Interface Sci., 172:278-288, 1995.

23. O. Vizika and J. M. Lombard. Wettability and spreading: Two key parameters in oil recovery with three-phase gravity drainage. SPE Reservoir Engng., 11:54-60, 1996.

24. V. Mani and K. K. Mohanty. Effect of spreading coefficient on three-phase flow in porous media. J. Colloid Interface Sci., 187:45, 1997.

25. M. J. Blunt. Note: Constraints on contact angles for multiple phase in thermodynamic equilibrium. J. Colloid Interface Sci., 239:281-282, 2001.

26. M. H. Hui and M. J. Blunt. Pore-scale modeling of three-phase flow and the effects of wettability. SPE 59309. Proceeding of the 2000 SPE/DOE Improved Oil Recovery Symposium, Tulsa, Oklahoma, 2000.

27. M. H. Hui and M. J. Blunt. Effects of wettability on three-phase flow in porous media. J. Phys. Chem. B, 104:3833-3845, 2000.

28. P. E. Øren and W. V. Pinczewski. Fluid distribution and pore-scale displacement mechanisms in drainage dominated three-phase flow. Trans. Porous Media, 20:105-33, 1995.

29. A. A. Keller, M. J. Blunt, and P. V. Roberts. Micromodel observation of the role of oil layers in three phase flow. Trans. Porous Media, 20:105-133, 1997.

30. M. I. J. van Dijke, K. S. Sorbie, M. Sohrabi, D. Tehrani, and A. Danesh. Three-phase flow in wag processes in mixed-wet porous media: pore-scale network simulations and comparison with micromodel experiments. SPE 75192. Proceedings of the 13th SPE/DOE Symposium on Improved Oil Recovery, Tulsa, Oklahoma, 2002. 